\title{
Short-segment cement-augmented fixation in open separation surgery of metastatic epidural spinal cord compression: initial experience
}

\author{
William C. Newman, MD, ${ }^{1}$ Anubhav G. Amin, MD, ${ }^{1,2}$ Jemma Villavieja, BS, ${ }^{1}$ llya Laufer, MD, MS, ${ }^{1,2}$ \\ Mark H. Bilsky, MD, ${ }^{1,2}$ and Ori Barzilai, MD'1
}

1Department of Neurological Surgery, Memorial Sloan Kettering Cancer Center; and 2Department of Neurological Surgery, Weill Cornell Medical College, NewYork-Presbyterian Hospital, New York, New York

\begin{abstract}
OBJECTIVE High-grade metastatic epidural spinal cord compression from radioresistant tumor histologies is often treated with separation surgery and adjuvant stereotactic body radiation therapy. Historically, long-segment fixation is performed during separation surgery with posterior transpedicular fixation of a minimum of 2 spinal levels superior and inferior to the decompression. Previous experience with minimal access surgery techniques and percutaneous stabilization have highlighted reduced morbidity as an advantage to the use of shorter fixation constructs. Cement augmentation of pedicle screws is an attractive option for enhanced stabilization while performing shorter fixation. Herein, the authors describe their initial experience of open separation surgery using short-segment cement-augmented pedicle screw fixation for spinal reconstruction.
\end{abstract}

METHODS The authors performed a retrospective chart review of patients undergoing open (i.e., nonpercutaneous, minimal access surgery) separation surgery for high-grade epidural spinal cord compression using cement-augmented pedicle screws at single levels adjacent to the decompression level(s). Patient demographics, treatment data, operative complications, and short-term radiographic outcomes were evaluated.

RESULTS Overall, 44 patients met inclusion criteria with radiographic follow-up at a mean of 8.5 months. Involved levels included 19 thoracic, 5 thoracolumbar, and 20 lumbar. Cement augmentation through fenestrated pedicle screws was performed in 30 patients, and a vertebroplasty-type approach was used in the remaining 14 patients to augment screw purchase. One (2\%) patient required an operative revision for a hardware complication. Three $(7 \%)$ nonoperative radiographic hardware complications occurred, including 1 pathologic fracture at the index level causing progressive kyphosis and 2 incidences of haloing around a single screw. There were 2 wound complications that were managed conservatively without operative intervention. No cement-related complications occurred.

CONCLUSIONS Open posterolateral decompression utilizing short-segment cement-augmented pedicle screws is a viable alternative to long-segment instrumentation for reconstruction following separation surgery for metastatic spine tumors. Studies with longer follow-up are needed to determine the rates of delayed complications and the durability of these outcomes.

https://thejns.org/doi/abs/10.3171/2021.2.FOCUS217

KEYWORDS cement augmentation; epidural spinal cord compression; metastatic disease; posterolateral decompression; spinal fixation

$\mathrm{M}$ ETASTASES to the spinal column are common in malignant disease and can manifest with a combination of pain, mechanical instability, and neurological deficits. ${ }^{1-3}$ The treatment of spinal metastases is palliative with the goal of alleviating pain, preserving or restoring neurological function, maintaining spinal stability, and achieving local tumor control while minimizing patient morbidity. To this end, the management of spinal metastatic disease is dependent on the tumor histology, the extent of epidural spinal cord compression (ESCC), patient symptomatology, and bony integrity. ${ }^{4}$ Spine stereotactic body radiation therapy (SBRT) has demonstrated durable local control of tumors previously considered radioresistant when used as either definitive therapy or a

ABBREVIATIONS ASIA = American Spinal Injury Association; ECOG = Eastern Cooperative Oncology Group; ESCC = epidural spinal cord compression; MRC = Medical Research Council; SBRT = stereotactic body radiation therapy.

SUBMITTED January 1, 2021. ACCEPTED February 23, 2021.

INCLUDE WHEN CITING DOI: 10.3171/2021.2.FOCUS217. 

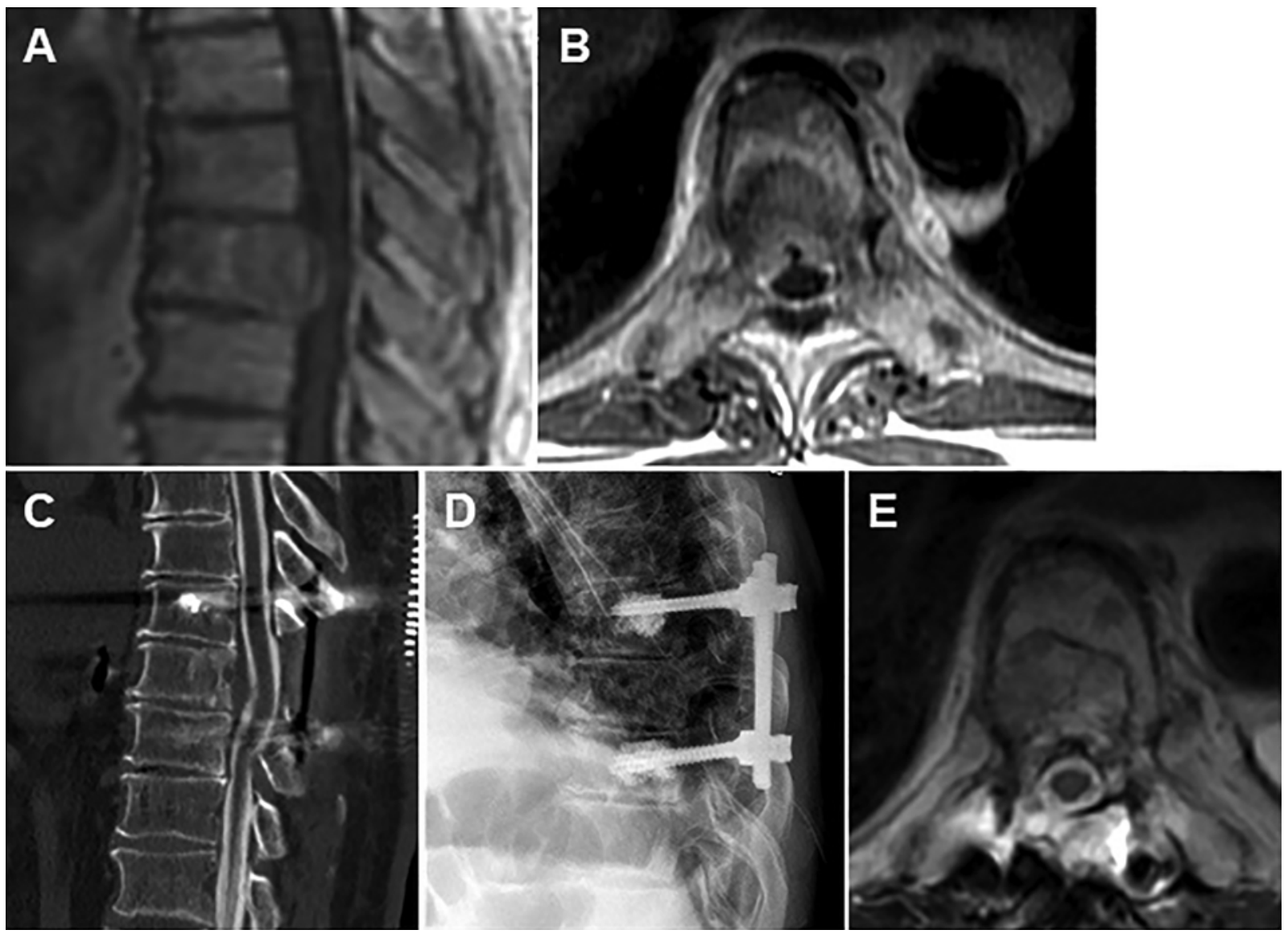

FIG. 1. Images obtained in a 78-year-old man with a history of metastatic thyroid cancer who presented with asymptomatic grade 2 ESCC. A and B: Sagittal (A) and axial (B) postcontrast T1-weighted MR images demonstrating grade 2 ESCC. The patient underwent separation surgery with posterolateral decompression at and placement of T7-9 cement-augmented pedicle screw fixation. C: Sagittal CT myelogram obtained in the immediate postoperative period demonstrating good decompression with reconstitution of the thecal sac. D: Lateral radiograph obtained immediately postoperatively demonstrating short-segment hardware placement with cement augmentation. E: Axial T2-weighted MR image obtained 9 months after hybrid therapy demonstrating regression of the epidural tumor with no compression of the thecal sac.

postoperative adjuvant. ${ }^{5,6}$ Subsequently, the goal of surgical intervention has shifted from aggressive resection to circumferential decompression and reconstitution of the thecal sac, or "separation surgery," 7,8 creating an optimal target for SBRT (i.e., "hybrid therapy"). 9

In order to achieve the circumferential separation of tumor from neurological structures, complete removal of the posterior elements is required. Hence, even patients with no clear signs of spinal instability preoperatively will require posterior segmental fixation because, in the process of removing the posterior elements, spinal stability is compromised. ${ }^{4,10}$ Thus, posterior segmental fixation is a vital component of this treatment. Recent technological advances, including fenestrated and expandable pedicle screws, have demonstrated superior biomechanical properties and have suggested a reduction in the number of hardware failures when utilized..$^{11,12}$ As a result of these advances, we have transitioned to the use of cement augmentation for percutaneous stabilization with low rates of symptomatic hardware failure requiring revision surgery. ${ }^{13,14}$ This experience has led us to explore the possi- bility of minimizing the extent of posterior segmental fixation for open decompression with short-segment cement-augmented constructs (Fig. 1). Herein, we review our initial experience with these constructs.

\section{Methods}

\section{Design}

This was a retrospective, single-center observational study at a tertiary cancer center. The local institutional review board approved this study, and informed consent was obtained. Patient clinical and radiographic information was obtained preoperatively, at 3 weeks immediately postoperatively, at 3-month intervals until 1 year postoperatively, and then yearly until the conclusion of follow-up. Signs of symptomatic or asymptomatic radiographic hardware failure were the primary endpoint. At each clinical follow-up point, we also obtained American Spinal Injury Association (ASIA) impairment scale scores, Eastern Cooperative Oncology Group (ECOG) performance status scores, and Medical Research Council (MRC) muscle strength scores. 


\section{Patient Population}

The records of patients who underwent open posterolateral decompression and resection of epidural metastases in the thoracic or lumbar spine with cement-augmented pedicle screw fixation at 1 level above and 1 level below the index level(s) (i.e., short segment) were screened. Inclusion criteria were imaging confirmation of metastatic epidural disease, with or without clinical symptoms of mechanical instability, in patients who underwent open separation surgery with short-segment cement-augmented pedicle screw constructs. Patients who had a segmental kyphosis or deformity from vertebral body fracture were included for consideration. Patients were excluded from consideration if fixation crossed the cervicothoracic junction or adequate fluoroscopic imaging could not be obtained for safe cement delivery. Additionally, patients who required stabilization over multilevel discontiguous disease were not considered for short-segment fixation. Patients who underwent percutaneous stabilization and/ or minimal access tubular decompression, or those who did not require decompression, were excluded from this analysis.

\section{Treatment}

All patients included in this study underwent posterolateral decompression with cement-augmented posterior instrumented fixation. In this study, cement augmentation included either the use of fenestrated screws or direct vertebroplasty-type injection of cement through the tapped screw track. Over the course of the study, there was a transition from cement delivery via vertebroplasty-type approach to fenestrated screws as they became available.

All surgeries were performed by one of 3 surgeons (M.H.B., I.L., or O.B.). A detailed method of performing separation surgery has been described elsewhere ${ }^{15}$ and was performed in the cases included in this series, with the only variation being the shorter constructs and the cement-augmentation techniques.

In brief, surgery consists of a linear midline incision and subperiosteal dissection of paraspinal muscles and ligament to expose the transverse processes at each level. Short-segment pedicle screw fixation is placed using neuronavigation and cement augmentation using fluoroscopy. Segmental kyphosis and fracture deformities are fixated in situ without corrective maneuvers. The bony decompression is tailored to the specific exposure necessary for safe tumor resection. The laminae, medial pedicle(s), and facet joint(s) are resected with a 3-mm matchstick burr. Posterolateral tumor resection is initiated from normal dural planes with nerve root sparing. A posterior partial corpectomy is performed, followed by resection of the posterior longitudinal ligament to affect a margin on the anterior dura mater, thus creating a circumferential decompression. Cement augmentation is used to fill large vertebral body defects.

\section{Data Analysis}

This was a retrospective, observational study utilizing descriptive analysis of patient clinical and radiographic outcomes.

\section{Results \\ Preoperative Demographics}

Overall, 44 patients who underwent separation surgery for metastatic epidural disease with short-segment cement-augmented constructs and had $>1$ month of radiographic follow-up were included in this retrospective review. The mean age at the time of surgery was 63 years, and $22(50 \%)$ patients were female.

Thirty-three $(75 \%)$ patients presented with preoperative high-grade ESCC grade 2 or 3 . The Spine Instability Neoplastic Score (SINS) ranged from 2 to 18, and patients were classified as follows: 9 (20\%) stable, 29 (66\%) potentially unstable, and 6 (14\%) unstable. The most common tumor histologies treated were non-small cell lung $(\mathrm{n}=$ $10,23 \%)$, prostate $(n=6,13 \%)$, colorectal $(n=5,11 \%)$, and breast $(\mathrm{n}=4,9 \%)$ carcinoma. Preoperatively, most patients had ASIA grade $\mathrm{E}(\mathrm{n}=34,77 \%)$, had an MRC score of 5 $(\mathrm{n}=34,77 \%)$, and an ECOG score of $1(\mathrm{n}=26,59 \%)$ or 2 $(\mathrm{n}=12,27 \%)$ (Table 1$)$.

\section{Operative Details}

Of the operations in 44 patients who underwent open short-segment cement-augmented fixation, 19 (43\%) were performed in the thoracic spine, $20(45 \%)$ were in the lumbar spine, and $5(11 \%)$ crossed the thoracolumbar junction (Table 2). No patients had previous surgery at the index level. Most patients underwent a 2-level posterolateral resection of the epidural tumor $(\mathrm{n}=36,81 \%)$, with 5 patients $(11 \%)$ receiving vertebral body cement augmentation at the index level. Fenestrated screws were used in 30 patients (68\%), and vertebroplasty via the cannulated pedicle tracks was performed in the remaining 14 patients, immediately followed by pedicle screw placement into the vertebral body. The mean estimated blood loss was 330 $\mathrm{mL}$, with a mean operative duration of 161 minutes. The average hospital length of stay was 4.7 days.

For patients receiving postoperative radiation therapy, the average time to treatment was 20 days, with a median time of 18 days. All patients received SBRT with doses ranging from $24 \mathrm{~Gy}$ in a single fraction to $40 \mathrm{~Gy}$ in 5 fractions.

\section{Radiographic Outcomes}

The mean duration of radiographic follow-up was 8.5 months. Overall, $1(2 \%)$ patient required an operative intervention for revision of the pedicle-screw construct. This patient initially underwent a T1-3 posterolateral decompression with cement-augmented fixation (Fig. 2). Three months after the initial surgery, the patient demonstrated symptomatic fractures of the bilateral T3 pedicle screws with progressive kyphosis for which he was taken back to the operating room for a T1-5 extension of instrumented fixation with cement augmentation (Fig. 2).

There were $3(7 \%)$ additional cases of asymptomatic radiographic findings at a mean of 3 months: 2 (4\%) patients had screw haloing at the top of the construct (both patients underwent L1-3 fixation), and 1 (2\%) patient had progressive kyphosis at the index level (L1) due to progression of a pathologic fracture. There were no cases of cementrelated complications (extravasation into the spinal canal 
TABLE 1. Patient demographics

\begin{tabular}{|c|c|}
\hline Variable & Value \\
\hline Total patients & $44(100)$ \\
\hline \multicolumn{2}{|l|}{ Age, yrs } \\
\hline Range & $37-83$ \\
\hline Mean & 63.7 \\
\hline Median & 63.2 \\
\hline \multicolumn{2}{|l|}{ Sex } \\
\hline Female & $22(50)$ \\
\hline Male & $22(50)$ \\
\hline \multicolumn{2}{|l|}{ Preop ESCC grade } \\
\hline 0 & $2(5)$ \\
\hline $1 \mathrm{~A}$ & $3(7)$ \\
\hline $1 \mathrm{~B}$ & $3(7)$ \\
\hline $1 \mathrm{C}$ & $3(7)$ \\
\hline 2 & $9(20)$ \\
\hline 3 & $24(55)$ \\
\hline \multicolumn{2}{|l|}{ Preop SINS } \\
\hline Range & $2-18$ \\
\hline Mean & 10 \\
\hline Median & 10 \\
\hline 0-6 (stable) & $9(20)$ \\
\hline 7-12 (potentially unstable) & $29(66)$ \\
\hline 13-18 (unstable) & $6(14)$ \\
\hline \multicolumn{2}{|l|}{ Preop ASIA grade } \\
\hline$E$ & $34(77)$ \\
\hline D & $5(11)$ \\
\hline C & $5(11)$ \\
\hline $\mathrm{B}$ & $0(0)$ \\
\hline A & $0(0)$ \\
\hline \multicolumn{2}{|l|}{ Preop ECOG score } \\
\hline 0 & $4(9)$ \\
\hline 1 & $26(59)$ \\
\hline 2 & $12(27)$ \\
\hline 3 & $2(5)$ \\
\hline \multicolumn{2}{|l|}{ Preop MRC score } \\
\hline 0 & $1(2)$ \\
\hline 1 & $0(0)$ \\
\hline 2 & $3(7)$ \\
\hline 3 & $1(2)$ \\
\hline 4 & $5(11)$ \\
\hline 5 & $34(77)$ \\
\hline Previous surgery at index site & $44(100)$ \\
\hline
\end{tabular}

SINS = Spine Instability Neoplastic Score.

Values represent the number of patients (\%) unless stated otherwise.

or pulmonary embolism) that required surgery or change in management.

\section{Clinical Outcomes}

The mean duration of clinical follow-up was 10.7 months. There were 18 deaths (41\%) during follow-up.
TABLE 2. Operative, clinical, and radiographic details

\begin{tabular}{|c|c|}
\hline Variable & Value \\
\hline \multicolumn{2}{|l|}{ Fixation location } \\
\hline Thoracic & $19(43)$ \\
\hline Thoracolumbar junction & $5(11)$ \\
\hline Lumbar & $20(45)$ \\
\hline \multicolumn{2}{|l|}{ Kyphoplasty at index level } \\
\hline Yes & $5(11)$ \\
\hline No & $39(89)$ \\
\hline \multicolumn{2}{|l|}{ Fenestrated screws } \\
\hline Yes & $30(68)$ \\
\hline No & $14(32)$ \\
\hline \multicolumn{2}{|l|}{ Laminectomy levels } \\
\hline 1 & $1(2)$ \\
\hline 2 & $36(81)$ \\
\hline 3 & $7(16)$ \\
\hline Mean estimated blood loss, $\mathrm{mL}$ & 330 \\
\hline Mean OR time, mins & 161 \\
\hline Mean length of stay, days & 4.7 \\
\hline$\geq 3$ mos clinical follow-up & 43 \\
\hline Mean length of clinical follow-up, days & 320 \\
\hline$\geq 3$ mos radiographic follow-up & $37(84)$ \\
\hline Mean radiographic follow-up, days & 257 \\
\hline Hardware failure requiring return to $\mathrm{OR}$ for revision & $1(2)$ \\
\hline Screw loosening (asymptomatic) & $2(4)$ \\
\hline Progressive kyphosis (asymptomatic) & $1(2)$ \\
\hline Wound complications & $2(4)$ \\
\hline Wound complications requiring return to $\mathrm{OR}$ & $0(0)$ \\
\hline Cement-related complication (PE, cord compression) & $0(0)$ \\
\hline
\end{tabular}

$\mathrm{OR}=$ operating room; $\mathrm{PE}=$ pulmonary embolism.

Values represent the number of patients (\%) unless stated otherwise.

Concerning functional outcomes, 1-month postoperative ECOG, ASIA, and MRC scores were available for 32 (73\%) patients. Most patients had no change (16 patients, $50 \%$ ) or improvement (13 patients, $40 \%$ ) in their ECOG score. The condition of $3(9 \%)$ patients worsened due to a progression of systemic disease. All patients had stable or improved 1-month ASIA and MRC grades.

In addition to hardware complications, 1 patient developed local cellulitis and 1 patient had a superficial wound dehiscence. Both wound complications were managed conservatively and did not require surgical intervention.

\section{Discussion}

In this preliminary experience, we performed 44 shortsegment cement-augmented posterolateral decompressions for metastatic epidural spinal cord compression, with only 1 case $(2 \%)$ of hardware failure that required surgery and 3 asymptomatic radiographic hardware-related issues at a median radiographic follow-up of 8.5 months. All patients had stable or improved ASIA and MRC scores at the 1-month follow-up. Three patients had a decline in ECOG 

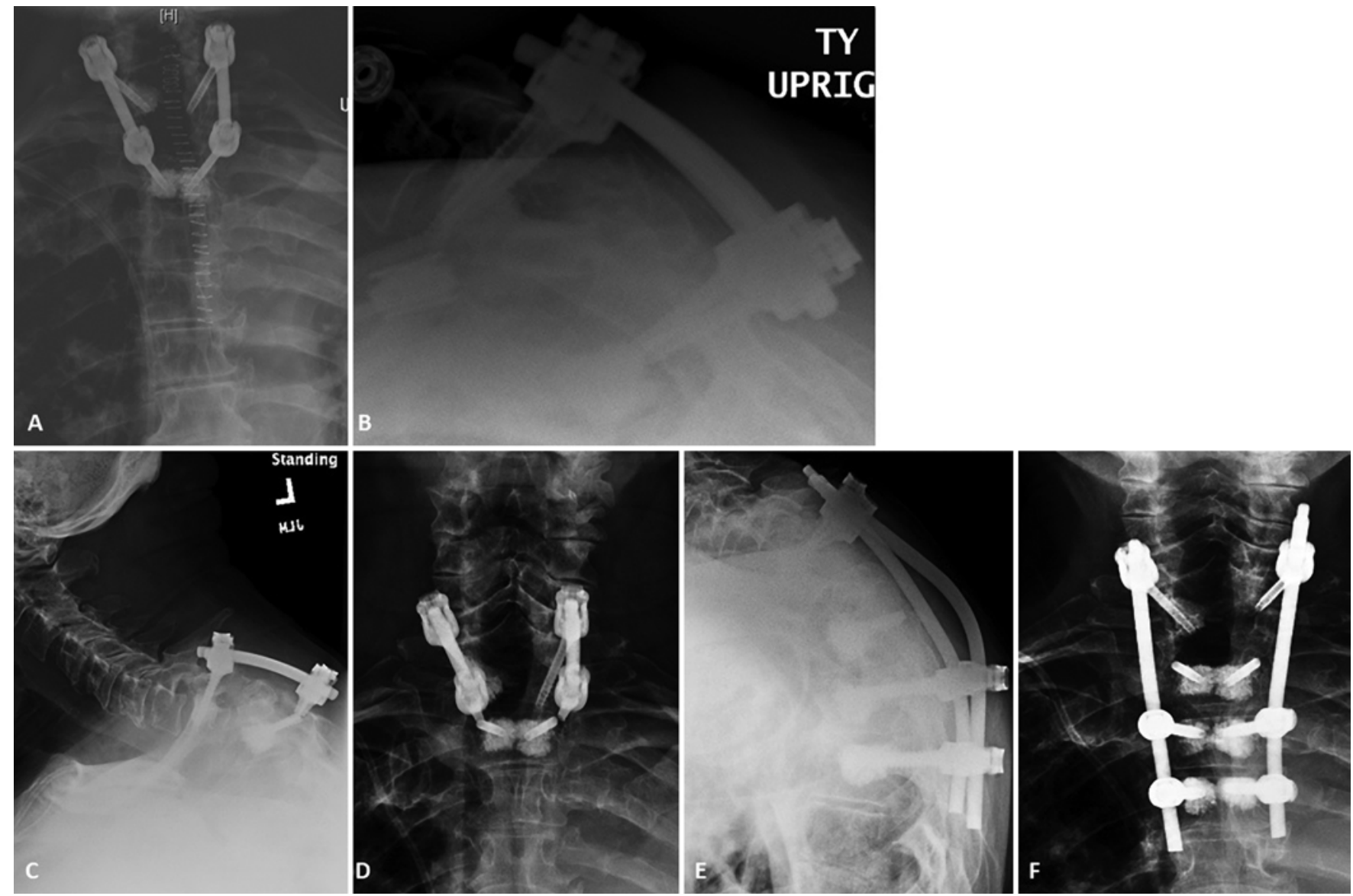

FIG. 2. Images obtained in a 61-year-old man with a history of non-small cell lung adenocarcinoma who presented with metastatic spread to his T2 vertebra with a lytic compression fracture and radicular pain. The patient underwent a T2 posterolateral decompression with placement of T1-3 cement-augmented pedicle screws. A and B: Anteroposterior (A) and lateral (B) radiographs obtained immediately postoperatively demonstrating cement-augmented pedicle screw placement at $\mathrm{T} 1$ and $\mathrm{T} 3$. C and D: At the 3-month follow-up, bilateral T3 screws were found to have fractured and kyphosis had worsened as seen on lateral (C) and anteroposterior (D) radiographs. The patient was subsequently taken back to the operating room for revision surgery. $\mathrm{E}$ and $\mathrm{F}$ : Postoperative lateral $(\mathrm{E})$ and anteroposterior $(\mathrm{F})$ radiographs obtained after revision demonstrating extension of the construct from T1 to T5 with retained distal portion of the cemented pedicle screw at T3. Nine months after revision surgery, the patient remained stable and his pain was well controlled.

performance score due to aggressive systemic disease progression.

Cancer patients are known to have poor bone quality due to a combination of osteolytic metastases, chemotherapy, radiation therapy, osteoporosis, and other comorbidities. An additional observation is that tumor progression frequently occurs at spine segments directly adjacent to the index level. In order to overcome these issues, instrumentation strategies for separation surgery have traditionally used long-segment constructs at a minimum of $2 \mathrm{lev-}$ els above and below the tumor-involved segments. ${ }^{16,17}$ As an adjunct to posterior fixation, tumor resection of $>50 \%$ of the vertebral body are additionally reconstructed with open cement augmentation at the index level. A review of 318 patients undergoing separation surgery using long-instrumented reconstructions with selective vertebral body cement augmentation demonstrated a $2.8 \%$ rate of symptomatic hardware failure requiring revision at a median follow-up of 13.3 months. ${ }^{18}$
In order to overcome the problem of poor bone quality, a number of strategies are being explored to increase the pullout strength and to minimize the need for longer-segment fixation, including expandable screws ${ }^{19}$ and cement augmentation of pedicle screws. ${ }^{12,13,20-22}$ These strategies have been explored in cases of trauma, osteoporosis, and percutaneous stabilization for pathologic fractures. Both biomechanical and clinical studies prompted us to explore the use of short-segment cement-augmented pedicle screws for reconstruction in open separation surgery.

Regarding the 4 patients who had radiographic evidence of fixation hardware failure, all involved constructs were at junctional spine segments. Both patients with radiographic evidence of screw loosening underwent L1-3 fixation and subsequently developed haloing around one of the L1 screws and an associated fracture. The patient with radiographic progression of kyphosis underwent T12-L2 fixation and had worsening L1 vertebral body fracture. As mentioned previously, the patient with clinically relevant 
hardware failure initially underwent T1-3 fixation and had progressive kyphosis with bilateral T3 screw fractures requiring extension of fixation to T5. The location of these failures at or near junction levels is consistent with findings from the non-cement-augmented treatment of thoracolumbar burst fractures with short-segment constructs where early hardware failures at junctional segments were found. ${ }^{23,24}$ Regarding the use of short-segment cementaugmented constructs near junctional levels, cadaveric data has suggested that nonaugmented multilevel fixation is biomechanically superior to short segments with cement augmentation, but this superiority is largely outside the realm of physiological loading. ${ }^{12}$ Additionally, Shin et al. demonstrated that, with the use of bracing and mobility restrictions, these junctional failure rates can be improved. ${ }^{25}$ Taken together, these findings may suggest that junctional interventions may warrant the use of additional levels of fixation or prophylactic bracing with mobility restrictions, yet more robust patient outcomes data are needed.

The ability to obtain wide circumferential decompression without having to instrument multiple levels above and below the index level decreases operative time, blood loss, and patient morbidity. The use of cement augmentation has been shown to result in a nearly twofold increase in pullout strength compared with nonaugmented screws. ${ }^{26-28}$ Within the arena of cement augmentation, there are three different design choices for screws: solid screws with cement augmentation via vertebroplasty, partially fenestrated screws with distal openings for cement extrusion, and fully fenestrated screws. While limited cadaveric data have suggested that partially fenestrated screws with cement augmentation have higher pullout strengths than solid screws augmented with the vertebroplasty technique, there appears to be no significant difference between fully and partially fenestrated screws. ${ }^{11}$ Additionally, that same study found no significant difference in removal torque forces between the three groups.

Although much of the literature discusses posterior pullout strength, posterior pullout is not the most common clinical mechanism for failure. Axial load testing more closely resembles physiological fatigue conditions, and, while cement-augmented screws are more resistant to failure than nonaugmented screws, multilevel nonaugmented constructs are significantly more resistant to failure. ${ }^{12}$ However, it is worth noting that while the fatigue force of short-segment cement-augmented constructs is less than that of multilevel nonaugmented constructs, the short-segment cement-augmented constructs are able to withstand most fatigue forces within physiological ranges.

Whereas biomechanical studies may have suggested that the use of fenestrated screws is superior to vertebroplasty with subsequent screw placement, ${ }^{11}$ we did not find this to be the case in our initial series. Of our 4 radiographic complications, fenestrated screws were used in 3 patients and cement vertebroplasty with subsequent solid screw placement was used in the remaining patient. While more patients are needed to determine if there are any differences in outcomes between short-segment cement augmentation with fenestrated versus solid screws, our initial experience seems to suggest that either may be used with good outcomes.

While we have previously published on the use of short- segment percutaneous pedicle screw fixation with cement augmentation for tumor-induced spinal instability with associated vertebral body fractures,${ }^{14}$ to our knowledge, the use of short-segment cement-augmented constructs in patients requiring open posterolateral decompression for separation surgery has not been described. The ability to utilize short cement-augmented constructs to maintain spinal stability while performing separation is appealing for cancer patients because of the ability to limit blood loss, operative time, and potential patient morbidity compared with longer-segment constructs in a category of patients with multiple comorbidities and variable degrees of frailty. Furthermore, this strategy may be beneficial for surgeons who do not perform minimally invasive spine surgery. Additionally, our mean length of stay of 4.7 days was similar to that of our earlier cohort of patients undergoing percutaneous pedicle screw fixation with percutaneous cement augmentation, ${ }^{13}$ which is another indicator of increased toleration by cancer patients of these less invasive, short-construct surgeries. Maximizing spinal stability while minimizing operative morbidity is especially critical when considered in context of the limited survival rate of our patients after intervention, which was 10.7 months in this series.

\section{Study Limitations}

Whereas this series represents a limited initial experience, several additional limitations should be noted. There are inherent flaws from the retrospective nature of analysis. Moreover, this is a small series of 44 patients with a combination of cement augmentation via either a fenestrated or vertebroplasty-type technique. A larger group would allow for better analysis of the impact of junctional location, as well as fenestrated versus solid screws on hardware failure. Furthermore, the short duration of our follow-up limits our ability to know if we would see more hardware failures at a delayed point or if the asymptomatic radiographic complications that we observed would progress to become symptomatic.

\section{Conclusions}

Based on our initial findings, for patients with metastatic spinal disease with limited life expectancy, shortsegment cement-augmented pedicle screws used as a reconstructive strategy for open separation surgery provides durable stabilization compared with long constructs that have been traditionally used. All cases of radiographic and clinical hardware failure involved fixation constructs at junctional levels. Studies with longer follow-up, incorporation of patient-reported outcomes, and specific attention to the utilization of this technique at or near the junctional spine are warranted.

\section{Acknowledgments}

This study was supported in part by the National Institutes of Health/National Cancer Institute Memorial Sloan Kettering Cancer Center support grant no. P30 CA008748.

\section{References}

1. Bach F, Larsen BH, Rohde K, et al. Metastatic spinal cord 
compression. Occurrence, symptoms, clinical presentations and prognosis in 398 patients with spinal cord compression. Acta Neurochir (Wien). 1990;107(1-2):37-43.

2. Schmidt MH, Klimo P Jr, Vrionis FD. Metastatic spinal cord compression. J Natl Compr Canc Netw. 2005;3(5):711-719.

3. Wong DA, Fornasier VL, MacNab I. Spinal metastases: the obvious, the occult, and the impostors. Spine (Phila Pa 1976). 1990;15(1):1-4.

4. Newman WC, Laufer I, Bilsky MH. Neurologic, oncologic, mechanical, and systemic and other decision frameworks for spinal disease. Neurosurg Clin N Am. 2020;31(2):151-166.

5. Yamada Y, Katsoulakis E, Laufer I, et al. The impact of histology and delivered dose on local control of spinal metastases treated with stereotactic radiosurgery. Neurosurg Focus. 2017;42(1):E6.

6. Laufer I, Iorgulescu JB, Chapman T, et al. Local disease control for spinal metastases following "separation surgery" and adjuvant hypofractionated or high-dose single-fraction stereotactic radiosurgery: outcome analysis in 186 patients. $J$ Neurosurg Spine. 2013;18(3):207-214.

7. Greco C, Pares O, Pimentel N, et al. Spinal metastases: from conventional fractionated radiotherapy to single-dose SBRT. Rep Pract Oncol Radiother. 2015;20(6):454-463.

8. Moulding HD, Elder JB, Lis E, et al. Local disease control after decompressive surgery and adjuvant high-dose singlefraction radiosurgery for spine metastases. J Neurosurg Spine. 2010;13(1):87-93.

9. Barzilai O, Amato MK, McLaughlin L, et al. Hybrid surgeryradiosurgery therapy for metastatic epidural spinal cord compression: a prospective evaluation using patient-reported outcomes. Neurooncol Pract. 2018;5(2):104-113.

10. Fisher CG, DiPaola CP, Ryken TC, et al. A novel classification system for spinal instability in neoplastic disease: an evidence-based approach and expert consensus from the Spine Oncology Study Group. Spine (Phila Pa 1976). 2010; 35(22):E1221-E1229.

11. Choma TJ, Pfeiffer FM, Swope RW, Hirner JP. Pedicle screw design and cement augmentation in osteoporotic vertebrae: effects of fenestrations and cement viscosity on fixation and extraction. Spine (Phila Pa 1976). 2012;37(26):E1628-E1632.

12. Weiser L, Dreimann M, Huber G, et al. Cement augmentation versus extended dorsal instrumentation in the treatment of osteoporotic vertebral fractures: a biomechanical comparison. Bone Joint J. 2016;98-B(8):1099-1105.

13. Barzilai O, McLaughlin L, Lis E, et al. Utility of cement augmentation via percutaneous fenestrated pedicle screws for stabilization of cancer-related spinal instability. Oper Neurosurg (Hagerstown). 2019;16(5):593-599.

14. Moussazadeh N, Rubin DG, McLaughlin L, et al. Shortsegment percutaneous pedicle screw fixation with cement augmentation for tumor-induced spinal instability. Spine J. 2015;15(7):1609-1617.

15. Barzilai O, Laufer I, Robin A, et al. Hybrid therapy for metastatic epidural spinal cord compression: technique for separation surgery and spine radiosurgery. Oper Neurosurg (Hagerstown). 2019;16(3):310-318.

16. Kumar N, Malhotra R, Maharajan K, et al. Metastatic spine tumor surgery: a comparative study of minimally invasive approach using percutaneous pedicle screws fixation versus open approach. Clin Spine Surg. 2017;30(8):E1015-E1021.

17. Yang Z, Yang Y, Zhang Y, et al. Minimal access versus open spinal surgery in treating painful spine metastasis: a systematic review. World J Surg Oncol. 2015;13:68.

18. Amankulor NM, Xu R, Iorgulescu JB, et al. The incidence and patterns of hardware failure after separation surgery in patients with spinal metastatic tumors. Spine J. 2014;14(9): $1850-1859$.
19. Gazzeri R, Roperto R, Fiore C. Surgical treatment of degenerative and traumatic spinal diseases with expandable screws in patients with osteoporosis: 2-year follow-up clinical study. J Neurosurg Spine. 2016;25(5):610-619.

20. Becker S, Chavanne A, Spitaler R, et al. Assessment of different screw augmentation techniques and screw designs in osteoporotic spines. Eur Spine J. 2008;17(11):1462-1469.

21. Chen LH, Tai CL, Lai PL, et al. Pullout strength for cannulated pedicle screws with bone cement augmentation in severely osteoporotic bone: influences of radial hole and pilot hole tapping. Clin Biomech (Bristol, Avon). 2009;24(8):613-618.

22. Elder BD, Lo SF, Holmes C, et al. The biomechanics of pedicle screw augmentation with cement. Spine J. 2015;15(6): 1432-1445.

23. Alanay A, Acaroglu E, Yazici M, et al. Short-segment pedicle instrumentation of thoracolumbar burst fractures: does transpedicular intracorporeal grafting prevent early failure? Spine (Phila Pa 1976). 2001;26(2):213-217.

24. McLain RF, Sparling E, Benson DR. Early failure of shortsegment pedicle instrumentation for thoracolumbar fractures. A preliminary report. J Bone Joint Surg Am. 1993;75(2): 162-167.

25. Shin TS, Kim HW, Park KS, et al. Short-segment pedicle instrumentation of thoracolumbar burst-compression fractures; short term follow-up results. J Korean Neurosurg Soc. 2007; 42(4):265-270.

26. Bostelmann R, Keiler A, Steiger HJ, et al. Effect of augmentation techniques on the failure of pedicle screws under cranio-caudal cyclic loading. Eur Spine J. 2017;26(1):181-188.

27. Chang MC, Kao HC, Ying SH, Liu CL. Polymethylmethacrylate augmentation of cannulated pedicle screws for fixation in osteoporotic spines and comparison of its clinical results and biomechanical characteristics with the needle injection method. J Spinal Disord Tech. 2013;26(6):305-315.

28. Sawakami K, Yamazaki A, Ishikawa S, et al. Polymethylmethacrylate augmentation of pedicle screws increases the initial fixation in osteoporotic spine patients. J Spinal Disord Tech. 2012;25(2):E28-E35.

\section{Disclosures}

Dr. Laufer: consultant for Medtronic, DePuy Synthes, and Brainlab; and royalties from Globus and Spine Wave. Dr. Bilsky: patent holder with DePuy Synthes and royalties from Globus.

\section{Author Contributions}

Conception and design: Barzilai. Acquisition of data: Newman, Amin, Villavieja. Analysis and interpretation of data: Barzilai, Newman, Amin, Bilsky. Drafting the article: Barzilai, Newman, Amin, Bilsky. Critically revising the article: Barzilai, Amin, Laufer, Bilsky. Reviewed submitted version of manuscript: all authors. Approved the final version of the manuscript on behalf of all authors: Barzilai. Study supervision: Barzilai.

\section{Supplemental Information \\ Current Affiliations}

Dr. Newman: Department of Neurological Surgery, Louisiana State University Health Sciences Center, Shreveport, Louisiana.

\section{Correspondence}

Ori Barzilai: Memorial Sloan Kettering Cancer Center, New York, NY.barzilaio@mskcc.org. 